Vol. 47 (1993) [101-118]

\title{
CRITICAL POINT THEOREMS WITH RELAXED BOUNDARY CONDITION AND APPLICATIONS
}

\author{
YIHONG DU
}

\begin{abstract}
This paper is a sequel to a recent paper by the author in this journal. We prove some variants of the min-max type critical point theorems with relaxed boundary condition and then apply the abstract results to a semilinear elliptic boundary value problem.
\end{abstract}

\section{INTRODUCTION}

Whether the mountain pass type min-max critical point theorems hold true with the relaxed boundary condition has interested several authors. Suppose $I$ is a $C^{1}$ functional on a Hilbert space $E$ satisfying the (PS) condition, and $S$ and $\partial Q$ are linking sets in E. Roughly speaking, the min-max critical point theorems say that if there exist $\alpha>\beta$ such that

$$
I(x) \geqslant \alpha \text { on } S \text { and } I(x) \leqslant \beta \text { on } \partial Q,
$$

then I has a critical value $c, c \geqslant \alpha$.

We call (1.1) the boundary condition in the critical point theorems. By the relaxed boundary condition we mean that there exists $\alpha$ such that

$$
I(x) \geqslant \alpha \text { on } S \text { and } I(x) \leqslant \alpha \text { on } \theta Q .
$$

In the mountain pass case, this problem and some related problems were studied in $[10,11]$, and it was proved in [9] and [12] that the mountain pass theorem is true under the relaxed boundary condition. Further results were obtained via Elland's variational principle in [8] and [6]. In [5] and [7], it was proved that more general min-max critical point theorems remain true under the relaxed boundary condition as well. In [5], a new deformation lemma was used while [7] used Ekland's variational principle. Other interesting related problems were also discussed in [7].

Minimax critical point theorems with boundary condition (1.1) such as the famous Mountain Pass Theorem have been widely used in obtaining nontrivial solutions of, for

Received 20th January, 1992.

Copyright Clearance Centre, Inc. Serialfee code: 0004-9729/93 SA2.00+0.00. 
example, elliptic boundary value problems. Therefore it is natural to expect new, better results in the applications when, instead of (1.1), the relaxed boundary condition (1.2) is used. In this paper, we are going to make an attempt in this direction. We make use of the relaxed boundary condition to the resonance problem

$$
\left\{\begin{array}{lll}
-\Delta u & =\lambda_{k} u+p(x, u) & \text { in } \Omega \\
u & =0 & \text { on } \partial \Omega
\end{array}\right.
$$

where $\Omega$ is a bounded domain in $R^{n}$ with $\partial \Omega$ sufficiently smooth, $p \in C\left(\bar{\Omega} \times R^{1}, R^{1}\right)$ is locally Lipschitz continuous and $p(x, 0)=0,0<\lambda_{1}<\ldots<\lambda_{k}<\lambda_{k+1}<\ldots$ are the eigenvalues of the problem $-\Delta u=\lambda u$ with homogeneous Dirichlet boundary condition. We use $m\left(\lambda_{i}\right)$ to denote the multiplicity of $\lambda_{i}$.

For this purpose, we need to prove some variants of the known critical point theorems with relaxed boundary condition; in particular, we should allow the linking sets to be infinite dimensional while earlier results of this type essentially require $\partial Q$ to be finite dimensional. In order to be able to treat the case with an infinite dimensional $\partial Q$, we have to make some restrictions on the functional $I$. Actually, as in [2], we require $I^{\prime}$ to be a variant of the form "Identity operator-Compact operator". For this type of functionals, we can prove that, in the deformation lemmas, the deformation maps can be chosen from a special mapping class. And this enables us to treat the infinite dimensional linking case because suitable infinite dimensional sets can link subject to this special mapping class.

\section{Deformation Lemmas}

In this section, we always assume that $\mathrm{E}$ is a real Hilbert space. For convenience we collect some definitions first.

Definition 2.1: A nonempty set $\Sigma$ in $C([0,1] \times E, E)$ is called a deformation class if $\phi_{1} \circ \phi_{2} \in \Sigma$ whenever $\phi_{1}, \phi_{2} \in \Sigma$.

Here

$$
\phi_{1} \circ \phi_{2}(t, x)=\phi_{1}\left(t, \phi_{2}(t, x)\right) \text { for }(t, x) \in[0,1] \times E .
$$

Let

$$
\begin{aligned}
\Sigma^{\circ} & =\{\phi \in C([0,1] \times E, E): \phi(t, x)=\phi(x)\}, \\
\Sigma_{A}^{\circ} & =\left\{\phi \in \Sigma^{\circ}: \phi(x)=x \text { for } x \in A\right\}, \\
\Sigma_{h} & =\left\{\phi \in C([0,1] \times E, E): \phi_{t} \text { is a homeomorphism of } \mathrm{E} \text { onto } \mathrm{E}\right\} .
\end{aligned}
$$

Evidently $\Sigma^{\circ}, \Sigma_{A}^{\circ}$ and $\Sigma_{h}$ are all deformation classes.

Definition 2.2: Let $\Sigma$ be a deformation class, $Q \subset E$ be a Banach manifold with boundary $\partial Q, S \subset E$ be a closed set. If $\partial Q \cap S=\emptyset$ and $\phi_{t}(Q) \cap S \neq \emptyset$ for every 
$t \in[0,1]$ whenever $\phi \in \Sigma$ and $\phi_{t}(\partial Q) \cap S=\theta$ for every $t \in[0,1]$, then we say that $\partial Q$ and $\mathbf{S} \boldsymbol{\Sigma}$-link.

We easily see that $\partial Q$ and $S$ link in the sense of $[1,3]$ and $[5]$ if and only if $\partial Q$ and $S \Sigma_{Q Q}^{\circ}$-link.

Let

$$
\begin{gathered}
\Delta=\{w \in C([0,1] \times E, E): w \text { is compact }\}, \\
\Lambda=\left\{\alpha \in C\left([0,1] \times E, R^{1} \backslash\{0\}\right): \alpha(t, x) \text { and }(\alpha(t, x))^{-1}\right. \\
\text { are both bounded on bounded sets }\} \\
\Sigma^{*}=\{\phi: \phi(t, x)=\alpha(t, x)(x-w(t, x)), \alpha \in \Lambda, w \in \Delta, \\
\alpha(0, x)=1, w(0, x)=0\}
\end{gathered}
$$

A simple verification shows

LemMa 2.1. $\Sigma^{*}$ is a deformation class.

Now we give some examples of $\Sigma^{*}$-linking sets.

Lemma 2.2. Suppose $E=E_{1} \oplus E_{2}, e \in \partial B_{1} \cap E_{1}, R>r>0$,

$$
S=\delta B_{r} \cap E_{1}, Q=\{s e: 0 \leqslant s \leqslant R\} \oplus B_{R} \cap E_{2} .
$$

Then $S$ and $\partial Q \Sigma^{*}$-link.

Proof: For any $\phi \in \Sigma^{*}$ satisfying $\phi_{t}(\partial Q) \cap S=\emptyset$ for each $t \in[0,1]$ we are going to prove that $\phi_{t}(Q) \cap S \neq \emptyset$ for each $t \in[0,1]$, that is, for each $t \in[0,1]$, there is some $q_{t} \in Q$ such that

$$
\left\|P_{1} \phi_{t}\left(q_{t}\right)\right\|=r \text { and } P_{2} \phi_{t}\left(q_{t}\right)=0
$$

where $P_{1}: E \rightarrow E_{1}, P_{2}: E \rightarrow E_{2}$ are projection operators.

In fact, for $u \in E_{2}, s \in R^{1}$, set

$$
\psi_{t}(s e+u)=s e+u+\left(\left(\left\|P_{1} \phi_{t}(s e+u)\right\|-r-s\right) e-P_{2} w_{t}(s e+u)\right) ;
$$

clearly $\psi_{t}$ is a compact perturbation of the identity operator. Moreover, since $\phi_{t}(\partial Q) \cap$ $S=0$ for $t \in[0,1]$, we have $0 \notin \psi_{t}(\partial Q)$ for $t \in[0,1]$. Hence the Leray-Schauder degree $\operatorname{deg}\left(\psi_{t}, \operatorname{int}(Q), 0\right)$ is well defined and is independent of $t$. So we have

$$
\operatorname{deg}\left(\psi_{t}, \operatorname{int}(Q), 0\right)=\operatorname{deg}\left(\psi_{0}, \operatorname{int}(Q), 0\right)=\operatorname{deg}(s e+u-r e, \operatorname{int}(Q), 0)=1,
$$

which implies that there exists some $q_{t} \in Q$ such that $\psi_{t}\left(q_{t}\right)=0$, that is,

$$
\left\|P_{1} \phi_{t}\left(q_{t}\right)\right\|=r \text { and } P_{2} w_{t}\left(q_{t}\right)=P_{2} q_{t} \text {, }
$$

and so

$$
P_{2} \phi_{t}\left(q_{t}\right)=\alpha\left(t, q_{t}\right)\left(P_{2} q_{t}-P_{2} w_{t}\left(q_{t}\right)\right)=0 \text {. }
$$


This completes the proof.

Lemm 2.3. Suppose $E=E_{1} \oplus E_{2}, Q=B_{R} \cap E_{2}, q \in \operatorname{int}(Q), S=q+E_{1}$. Then $\partial Q$ and $S \Sigma^{*}$-link.

Proor: For $\phi \in \Sigma^{*}$ with $\phi_{t}(\partial Q) \cap S=0$ we are going to prove that there is some $q_{t} \in Q$ such that $P_{2} \phi_{t}\left(q_{t}\right)=q$.

For $u \in E_{2}, P_{2} \phi_{t}(u)=\alpha(t, u)\left(u-P_{2} w_{t}(u)\right)$, so it suffices to prove

$$
u-\left(P_{2} w_{t}(u)+q\left(\alpha(t, u)^{-1}\right)\right)=0
$$

has a solution in $Q$.

Since $\phi_{t}(\partial Q) \cap S=\emptyset$ for $t \in[0,1]$, we easily see

and hence

$$
\begin{aligned}
u- & \left(P_{2} w_{t}(u)+q\left(\alpha(t, u)^{-1}\right)\right) \neq 0 \text { for } t \in[0,1], u \in \partial Q \\
& \operatorname{deg}\left(u-P_{2} w_{t}(u)-q\left(\alpha(t, u)^{-1}\right), \operatorname{int}(Q), 0\right) \\
= & \operatorname{deg}\left(u-P_{2} w_{0}(u)-q\left(\alpha(0, u)^{-1}\right), \operatorname{int}(Q), 0\right) \\
= & \operatorname{deg}(u-q, \operatorname{int}(Q), 0)=1
\end{aligned}
$$

This shows that for each $t \in[0,1], u-\left(P_{2} w_{t}(u)+q\left(\alpha(t, u)^{-1}\right)\right)=0$ has at least one solution in $Q$. The proof is complete.

Now we are going to give some deformation lemmas. We shall say that $f \in$ $C^{1}\left(E, R^{1}\right)$ satisfies the (PS) condition on $A \subset E$, if $\left\{x_{n}\right\} \subset A,\left\{f\left(x_{n}\right)\right\}$ is bounded and $f^{\prime}\left(x_{n}\right) \rightarrow 0$ imply the existence of a convergent subsequence of $\left\{x_{n}\right\}$.

The functional $f$ is said to satisfy condition (C) if

$1^{\circ}$. f satisfies the (PS) condition on every bounded sets;

$2^{\circ}$. For any $c \in R^{1}$, there exist $\sigma, R, \alpha>0$ such that

$$
c-\sigma \leqslant f(u) \leqslant c+\sigma \text { and }\|u\| \geqslant R \text { imply }\left\|f^{\prime}(u)\right\| \geqslant \alpha /\|u\| \text {. }
$$

Lemma 2.4. Suppose $f \in C^{1}\left(E, R^{1}\right), c \in R^{1}$, and $A$ and $B$ are two closed sets in $E$. Moreover, suppose the following four conditions are satisfied:

$1^{\circ}$. $f(x) \geqslant c$ on $A$ and $f(x) \leqslant c$ on $B$;

2. $A \cap B=0, B \cap K_{c}=0$;

$3^{\circ}$. I satisfies the (PS) condition on some $\delta$-neighbourhood $N_{\delta}(B)$ of $B$;

$4^{\circ}$. $f^{\prime}(x)=j x-D x, j=1$ or $-1, D: E \rightarrow E$ is completely continuous. 
Then there exist $\varepsilon>0$ and $\eta \in \Sigma^{*}$ such that:

(i) For every $t \in[0,1], \eta_{t}$ is a homeomorphism of $E$ onto $E$;

(ii) $f(\eta(t, x)) \leqslant f(x)$ for $x \in E$ and $t \in[0,1]$;

(iii) $\eta(t, x)=x$ for $x \in A$ and $t \in[0,1]$;

(iv) $f(\eta(1, x)) \leqslant c-\varepsilon$ for $x \in B$;

(v) $\eta^{-1} \in \Sigma^{*}$, where we define $\eta^{-1}(t, x)=\eta_{t}^{-1}(x)$.

Proof: We prove the lemma by three steps.

Step 1. Denote $K=\left\{x \in E: f^{\prime}(x)=0\right\}$. Then there exists a locally Lipschitz continuous map $v: E \backslash K \rightarrow E$ such that

$$
\begin{gathered}
\|v(x)\|<2\left\|f^{\prime}(x)\right\|,\left(f^{\prime}(x), v(x)\right)>1 / 2\left\|f^{\prime}(x)\right\|^{2} \text { for } x \in E \backslash K, \\
D_{1} x=j x-v(x) \text { is completely continuous. }
\end{gathered}
$$

In fact, by a standard partition of unity argument we can find a locally Lipschitz continuous map $D_{1}: E \backslash K \rightarrow E$ such that

$$
\begin{gathered}
\left\|D x-D_{1} x\right\|<1 / 2\left\|f^{\prime}(x)\right\| \text { for } x \in E \backslash K, \\
D_{1}(\Omega) \subset \overline{c o}\left(D\left(N_{1}(\Omega)\right)\right) \text { for any } \Omega \subset E \backslash K, \text { where } \\
N_{1}(\Omega)=\{x \in E \backslash K: \operatorname{dist}(x, \Omega) \leqslant 1\} .
\end{gathered}
$$

Let $v(x)=j x-D_{1} x$. From (2.4) we see that (2.2) is true. Using (2.3) and a simple computation we know that (2.1) is satisfied.

Step 2. There exists a locally Lipschitz continuous map $\lambda: E \rightarrow R^{1}$ such that

$$
\begin{gathered}
0 \leqslant \lambda(x) \leqslant 1 \text { on } E, \\
\lambda(x)=0 \text { on some neighbourhood } \mathrm{N}(\mathrm{K}) \text { of } \mathrm{K},
\end{gathered}
$$

and that the unique solution

$$
\left\{\begin{array}{l}
d \eta / d t=-\lambda(\eta) v(\eta) \\
\eta(0, x)=x
\end{array}\right.
$$

is defined on all $R^{1} \times E$. Moreover, $\eta$ satisfies (i)-(iv) and

$$
\|\eta(t, x)-x\| \leqslant 1 \text { for } t \in[0,1] \text { and } x \in E .
$$

Here we define $\lambda(x) v(x)=0$ for $x \in K$. This is reasonable because $\lambda(x)$ satisfies (2.6).

The conclusions in this step follow from the proof of Lemma 1.1 in [5]. The only difference is that here we suppose $f$ satisfies the (PS) condition on $N_{\delta}(B)$ while in [5] 
$f$ is supposed to satisfy the (PS) condition on the whole space E. However it can be easily seen that our present assumption is enough for the proof.

Step 3. $\eta, \eta^{-1} \in \Sigma^{*}$.

Extend $\left.D_{1}\right|_{E \backslash N(K)}$ to a completely continuous map $D_{2}$ on $E$, and for $x \in E$ define $v_{1}(x)=j x-D_{2} x$. It follows from (2.6) that $\lambda(x) v(x)=\lambda(x) v_{1}(x)$ on $E$. Therefore

$$
\begin{aligned}
& d / d t(\eta(t, x))=-\lambda(\eta) v_{1}(\eta)=-\lambda(\eta)\left(j \eta-D_{2}(\eta)\right) \\
& d / d t\left(\eta(t, x) \exp \left(j \int_{0}^{t} \lambda(\eta(\tau, x)) d \tau\right)\right) \\
& =\lambda(\eta(t, x)) D_{2}(\eta(t, x)) \exp \left(j \int_{0}^{t} \lambda(\eta(\tau, x)) d \tau\right) .
\end{aligned}
$$

Let

$$
\alpha(t, x)=\exp \left(-j \int_{0}^{t} \lambda(\eta(\tau, x)) d \tau\right)
$$

$$
w(t, x)=\int_{0}^{t} \lambda(\eta(s, x)) D_{2}(\eta(s, x)) \exp \left(j \int_{0}^{s} \lambda(\eta(\tau, x)) d \tau\right) d s .
$$

Clearly

$$
\eta(t, x)=\alpha(t, x)(x+w(t, x)) \text { and } \alpha(0, x)=1, w(0, x)=0 .
$$

Hence to prove $\eta \in \Sigma^{*}$ we only need to check that $\alpha \in \Lambda$ and $w \in \Delta$.

$\alpha \in \Lambda$ is obvious by (2.5). Moreover we have

$$
0 \leqslant \lambda(\eta(t, x)) \exp \left(j \int_{0}^{t} \lambda(\eta(\tau, x)) d \tau\right) \leqslant e \text { for } t \in[0,1] .
$$

By (2.7) we have

$$
\eta([0,1] \times \Omega) \subset N_{1}(\Omega) \text { for any } \Omega \subset E .
$$

Now it follows from (2.8) and (2.9) that for $x \in \Omega, t \in[0,1]$

$$
w_{t}(x)=\int_{0}^{t} \lambda(\eta) \exp \left(j \int_{0}^{2} \lambda(\eta) d \tau\right) D_{2}(\eta) d s \in e \cdot \overline{c o}\left(D_{2}\left(N_{1}(\Omega)\right), 0\right) .
$$

Hence $w_{t}(\Omega) \subset e \cdot \overline{c o}\left(D_{2}\left(N_{1}(\Omega)\right), 0\right)$ for $t \in[0,1]$. Since $D_{2}$ is completely continuous, this shows that $w_{t}$ is compact. Moerover, by the definition of $w$ we easily see that $w$ is continuous and that its continuity with respect to $t$ is uniform for $x$ in bounded sets. This implies the complete continuity of $w$, and so $w \in \Delta$ and $\eta \in \Sigma^{*}$.

Since $\eta^{-1}(t, x)=\eta(-t, x)$, a similar argument shows $\eta^{-1} \in \Sigma^{*}$. The proof is complete.

Lemma 2.5. Suppose $f \in C^{1}\left(E, R^{1}\right)$ satisfies condition $(C), c \in R^{1}, N$ is a neighbourhood of $K_{c}, f^{\prime}(x)=j x-D x, j=1$ or -1 , and $D: E \rightarrow E$ is completely continuous. Then for any $\sigma>0$ we can find $\bar{\varepsilon}, \varepsilon, 0<\varepsilon<\bar{\varepsilon}<\sigma$, and $\eta \in \Sigma^{*}$ such that

(i) For each $t \in[0,1], \eta_{t}$ is a homeomorphism of $E$ onto $E$; 
(ii) $f(\eta(t, x)) \leqslant f(x)$ for $x \in E$ and $t \in[0,1]$;

(iii) $\eta(t, x)=x$ for $x \in E \backslash f^{-1}([c-\bar{\varepsilon}, c+\bar{\varepsilon}])$;

(iv) $f(\eta(1, x)) \leqslant c-\varepsilon$ for $x \in f^{-1}((-\infty, c+\varepsilon]) \backslash N$, here we suppose $N=\emptyset$ in case $K_{c}=\emptyset$;

(v) $\eta^{-1} \in \Sigma^{*}$.

Proof: By step 1 in the proof of Lemma 2.4 we know that there exists a locally Lipschitz continuous map $v: E \backslash K \rightarrow E$ satisfying (2.1) and (2.2). Let

$$
\phi(x)=v(x) /\|v(x)\|^{2} \text { for } x \in E \backslash K .
$$

Then $\phi: E \backslash K \rightarrow E$ is locally Lipschitz continuous and satisfies

$$
\|\phi(x)\| \leqslant 2 /\left\|f^{\prime}(x)\right\|,\left(f^{\prime}(x), \phi(x)\right) \geqslant 1 / 8, x \in E \backslash K .
$$

By (2.10) and a similar argument to that in the proof of [1, Theorem 1.3] we can show that there exists a function $\chi: E \rightarrow[0,1]$ such that

$1^{\circ} . \chi(x)=0$ on some neighbourhood $N(K)$ of $K$;

$2^{\circ}$. For

$$
V(x)= \begin{cases}-\chi(x) \phi(x) & \text { for } x \in E \backslash K \\ 0 & \text { for } x \in K\end{cases}
$$

there exist constants $k_{1}$ and $k_{2}$ such that $\|V(x)\| \leqslant k_{2}+k_{2}\|x\|$;

$3^{\circ}$. The unique solution $\eta(t, x)$ of the initial problem

$$
\left\{\begin{array}{l}
d \eta / d t=V(\eta) \\
\eta(0, x)=x
\end{array}\right.
$$

is defined on $R^{1} \times E$, it satisfies the requirements (i)-(iv) in our lemma and is bounded on bounded sets.

Now if we can prove that $\eta, \eta^{-1} \in \Sigma^{*}$, then we are done.

Since $\eta^{-1}(t, x)=\eta(-t, x)$, as in the proof of Lemma 2.4, we only prove $\eta \in \Sigma^{*}$. Extend $\left.D_{1}\right|_{E \backslash N(K)}$ to a completely continuous map on $E$ and denote it by $D_{2}$. Let

$$
\lambda(x)= \begin{cases}\chi(x) /\|v(x)\|^{2} & \text { for } x \in E \backslash K, \\ 0 & \text { for } x \in K .\end{cases}
$$

It is easy to see that $V(x)=-\lambda(x)\left(j x-D_{2} x\right)$ for all $x \in E$. Moreover, $\lambda: E \rightarrow R^{1}$ is continuous and

$$
0 \leqslant \lambda(x) \leqslant x(x)\|\phi(x)\|^{2} \leqslant\left(x(x)\|\phi(x)\|^{2}=\|V(x)\|^{2} \leqslant\left(k_{1}+k_{2}\|x\|\right)^{2} .\right.
$$


Now since $d \eta / d t=-\lambda(\eta)\left(j \eta-D_{2}(\eta)\right)$, since $D_{2}$ is completely continuous, and since $\lambda$ and $\eta$ are bounded on bounded sets, we can show as in Step 3 of the proof of Lemma 2.4 that $\eta \in \Sigma^{*}$. The proof is complete.

REMARK: In Lemma 2.4 and Lemma 2.5, if $f$ is a even functional and $A, B$ are symmetric with respect to 0 , then we can choose $\eta$ to be odd in $\boldsymbol{x}$.

After these preparations, we are ready to prove some critical point theorems with relaxed boundary conditions in the next section.

\section{Critical Point Theorems}

Throughout this section, we suppose $E$ is a real Hilbert space, $Q$ is a closed Hilbert manifold with boundary $\partial Q$, and $S$ is a closed set in $E$. We are going to give some critical point theorems which are variants of known results. In earlier results, when the linking sets are allowed to be infinite dimensional, the stronger boundary condition (1.1) is required (see for example $[2,12]$ ), and when the relaxed boundary condition is used, one of the linking sets $\partial Q$ should be finite dimensional (this is often implicit in the theorems) and such results are generally proved under the (PS) condition rather than the condition (C) (see $[5,6,7]$ and [9]). Our results in this section are improvements of earlier theorems so that they are more convenient in applications.

Theorem 3.1. Suppose $\partial Q$ and $S \Sigma^{*}$-link, $f \in C^{1}\left(E, R^{1}\right)$ satisfies the (PS) condition on $E, f^{\prime}(x)=j x-D x, j=1$ or -1 , and $D$ is completely continuous. Moreover, suppose $\sup _{Q} f(x)<+\infty$ and there exists some $\alpha \in R^{1}$ such that

$$
\begin{gathered}
f(x) \leqslant \alpha \text { on } \partial Q ; f(x) \geqslant \alpha \text { on } S . \\
c=\inf _{\phi \in \Gamma_{0}} \sup _{x \in Q} f(\phi(1, x)),
\end{gathered}
$$

where $\Gamma_{0}=\left\{\phi \in \Sigma^{*}: \phi(t, x)=x\right.$ for $\left.x \in \partial Q, t \in[0,1]\right\}$. Then we have

(i) $c \geqslant \alpha$,

(ii) $K_{c} \neq 0$,

(iii) $K_{c} \cap S \neq \emptyset$ in case $c=\alpha$.

PRoOF: Notice first that $\phi_{0}(t, x) \equiv x$ is an element of $\Gamma_{0}$, so $\Gamma_{0} \neq 0$. For any $\phi \in \Gamma_{0}$, since $\phi_{t}(\partial Q) \cap S=\partial Q \cap S=0$ and $\partial Q$ and $S \Sigma^{*}$-link, we have $\phi(1, Q) \cap S \neq 0$. Suppose $x_{0} \in Q$ is such that $\phi\left(1, x_{0}\right) \in S$. Then it follows from (3.1) that

$$
\sup _{Q} f(\phi(1, x)) \geqslant f\left(\phi\left(1, x_{0}\right)\right) \geqslant \alpha \text { for ang } \phi \in \Gamma_{0}
$$

This implies $c \geqslant \alpha$. 
If $c=\alpha$, it suffices to prove $K_{c} \cap S \neq \emptyset$. Suppose this is not the case. Then apply Lemma 2.4 to $-f$ with $A=\partial Q$ and $B=S$; we find $\varepsilon>0$ and $\eta \in \Sigma^{*}$ such that

$$
\eta^{-1} \in \Sigma^{*}, \eta(t, x)=x \text { on } \partial Q,-f(\eta(1, x)) \leqslant-c-\varepsilon \text { on } S .
$$

By the definition of $c$ we can choose $\phi_{0} \in \Gamma_{0}$ such that

$$
f\left(\phi_{0}(1, x)\right)<c+\varepsilon \text { on } Q
$$

Let $\phi_{1}(t, x)=\eta^{-1}\left(t, \phi_{0}(t, x)\right)$. From (3.2) and Lemma 2.1 we see that $\phi_{1} \in \Gamma_{0}$, and hence there is some $x_{0} \in Q$ such that $\phi_{1}\left(1, x_{0}\right) \in S$ because $\partial Q$ and $S \Sigma^{*}$-link. Now it follows from (3.2) and $\phi_{1}\left(1, x_{0}\right) \in S$ that

$$
f\left(\phi_{0}\left(1, x_{0}\right)\right)=f\left(\eta \circ \phi_{1}\left(1, x_{0}\right)\right) \geqslant c+\varepsilon,
$$

which contradicts (3.3). Therefore we must have $K_{c} \cap S \neq \emptyset$ when $c=\alpha$.

If $c>\alpha$, using Lemma 2.5 and a standard argument (see, for example, $[1,3]$ or [12]) one easily get $K_{c} \neq \emptyset$. The proof is complete.

THEOREM 3.2. In Theorem 3.1, if $S$ is bounded, then the condition that $f$ satisfies the (PS) condition can be replaced by $f$ satisfies condition (C).

PROOF: In this case the conditions of Lemma 2.4 and Lemma 2.5 are still met, so by checking the proof of Theorem 3.1 we arrive at the conclusion. The proof is complete.

The following two theorems are concerned with even functionals.

THEOREM 3.3. Suppose that $f \in C^{1}\left(E, R^{1}\right)$ is a even functional and satisfies condition $(C), E_{1}$ and $E_{2}$ are finite dimensional subspaces of $E$, and $n=\operatorname{dim} E_{1}<$ $m=\operatorname{dim} E_{2}$. Moreover, suppose $f(0)=0$ and there exist $r, R, 0<r<R$ such that

$$
f(x) \geqslant 0 \text { on } E_{1}^{\perp} \cap \partial B_{r}, f(x) \leqslant 0 \text { on } E_{2} \cap \partial B_{R} .
$$

Then $f$ has at least $m-n$ pairs of nonzero critical points.

Proof: We follow the ideas of Rabinowitz [12]. Let

$$
\Sigma=\{A \subset E \backslash\{0\}: A \text { is symmetric with respect to } 0\},
$$

and let $\gamma$ be the genus on $\Sigma$, that is, $\gamma(\emptyset)=0$; and for $A \in \Sigma$ and $A \neq \emptyset$, define $\gamma(A)=n$, where $n$ is the smallest integer such that there exists an odd map $\phi \in$ $C\left(A, R^{n} \backslash\{0\}\right)$; define $\gamma(A)=\infty$ if there exists no such $n$. 
Suppose that $\left\{e_{1}, \ldots, e_{m}\right\}$ is a basis for $E_{2}$, and define

$$
\begin{aligned}
E^{(k)} & =\operatorname{span}\left\{e_{1}, \ldots, e_{k}\right\}, k=1, \ldots, m . \\
D_{k} & =E^{(k)} \cap B_{R}, \\
G_{k} & =\left\{h \in C\left(D_{k}, E\right): h \text { is odd, and } h x=x \text { on } \partial D_{k}=E^{(k)} \cap \partial B_{R}\right\}, \\
\Gamma_{j} & =\left\{h\left(\overline{D_{k} \backslash Y}\right): k \geqslant j, h \in G_{k}, Y \in \Sigma \text { and } \gamma(Y) \leqslant k-j\right\} .
\end{aligned}
$$

It is proved in $\left[12\right.$, p.56] that $\Gamma_{j}(j=1, \ldots, m)$ has the following properties:

$1^{\circ} . \quad \Gamma_{j} \neq \emptyset, j=1, \ldots, m$;

2。. $\Gamma_{j+1} \subset \Gamma_{j}, j=1, \ldots, m-1$;

$3^{\circ}$. If $\phi \in C(E, E)$ is odd and $\phi(x)=x$ on $E_{2} \cap \partial B_{R}$, then

$$
\phi(B) \in \Gamma_{j} \text { for } B \in \Gamma_{j}, j=1, \ldots, m \text {; }
$$

$4^{\circ}$. If $B \in \Gamma_{j}, Z \in \Sigma$ and $\gamma(Z) \leqslant s<j$, then $\overline{B \backslash Z} \in \Gamma_{j-s}$.

Now we prove

$5^{\circ}$. If $j \geqslant n+1$ and $\Delta \in \Sigma$ is a compact set with $\gamma(\Delta) \leqslant j-n-1$, then

$$
B \cap\left(E_{1}^{\perp} \cap \partial B_{r} \backslash \Delta\right) \neq \emptyset \text { for any } B \in \Gamma_{j} .
$$

In fact, for $B \in \Gamma_{j}$, suppose $B=h\left(\overline{D_{k} \backslash Y}\right), k \geqslant j, h \in G_{k}, Y \in \Sigma$ and $\gamma(Y) \leqslant k-j$. Let

$$
\widehat{V}=\left\{x \in D_{k}: h(x) \in \operatorname{int}\left(B_{r}\right)\right\},
$$

and let $\mathrm{V}$ denote the component of $\widehat{V}$ containing 0 . Since $h\left(\partial D_{k}\right)=\partial D_{k}=E^{(k)} \cap \partial B_{R}$, $R>r$, we see that $h\left(\partial D_{k}\right) \cap B_{r}=\emptyset$, and hence it follows from $h(0)=0$ that $V$ is a symmetric bounded open neighbourhood of 0 in $E^{(k)}$, which implies $\gamma(\partial V)=$ $\operatorname{dim} E^{(k)}=k$. Evidently $h(\partial V) \subset \partial B_{r}$. Let

$$
\widetilde{D}=\left\{x \in D_{h}: h(x) \in \partial B_{r}\right\} .
$$

Then $\widetilde{D} \in \Sigma, \widetilde{D} \supset \partial V$ and so $\gamma(\tilde{D}) \geqslant \gamma(\partial V)=k$,

$$
\gamma(h(\widetilde{\widetilde{D} \backslash Y})) \geqslant \gamma(\widetilde{\widetilde{D} \backslash Y}) \geqslant \gamma(\tilde{D})-\gamma(Y) \geqslant j .
$$

If $h(\widetilde{\widetilde{D} \backslash Y}) \cap\left(E_{1}^{\perp} \cap \partial B_{\mathbf{r}} \backslash \Delta\right)=\emptyset$, let

$$
A=h(\widetilde{\widetilde{D} \backslash Y}) \backslash N(\Delta)
$$


where $N(\Delta)$ is an open neighbourhood of $\Delta$ such that $\gamma(\overline{N(\Delta)})=\gamma(\Delta)$; then $A \in \Sigma$ and $A \cap E_{1}^{\perp} \cap \partial B_{r}=0$. From the definition of $\widetilde{D}$ we see that $A \in \partial B_{r}$, and therefore $A \cap E_{1}^{\perp} \cap \partial B_{r}=\emptyset$ implies $0 \notin P_{1} A$, where $P_{1}$ is the projector of $E$ onto $E_{1}$. Identify $E_{1}$ with $R^{n}$; since $P_{1}: A \rightarrow P_{1} A$ is odd, we have $\gamma(A) \leqslant n$. Therefore

$$
n \geqslant \gamma(A) \geqslant \gamma(h(\widetilde{D} \backslash Y))-\gamma(\overline{N(\Delta)}) \geqslant \gamma(h(\widetilde{\widetilde{D} \backslash Y}))-j+n+1,
$$

that is, $\gamma(h(\widetilde{\widetilde{D} \backslash Y})) \leqslant j-1$, which contradicts (3.5). Thus we must have $h(\widetilde{\widetilde{D} \backslash Y}) \cap$ $\left(E_{1}^{\perp} \cap \partial B_{r} \backslash \Delta\right) \neq \emptyset, B \cap\left(E_{1}^{\perp} \cap \partial B_{r} \backslash \Delta\right) \neq \emptyset$.

For $j=n+1, \ldots, m$, let

$$
c_{j}=\inf _{B \in \Gamma_{j}} \sup _{\boldsymbol{x} \in \boldsymbol{B}} f(x)
$$

From $2^{\circ}, 5^{\circ}$ and (3.4) we find

$$
0 \leqslant c_{n+1} \leqslant \ldots \leqslant c_{m}
$$

To prove that $f$ has at least $m-n$ pairs of nonzero critical points, it suffices to prove the following two assertions:

$$
\begin{aligned}
& 6^{\circ} . \text { If } c_{n+1}=\ldots=c_{n+k}=0,1 \leqslant k \leqslant m-n, \text { then } \gamma\left(K_{0} \cap E_{1}^{\perp} \cap \partial B_{r}\right) \geqslant k ; \\
& 7^{\circ} . \text { If } c_{n+k_{1}}=\ldots=c_{n+k_{2}}=c>0,1 \leqslant k_{1} \leqslant k_{2} \leqslant m-n, \text { then } \gamma\left(K_{c}\right) \geqslant \\
& k_{2}-k_{1}+1 .
\end{aligned}
$$

The proof of $7^{\circ}$ is standard (see [12] and use [1, Theorem 1.3] for even functionals), so we only prove $6^{\circ}$.

Suppose $c_{n+1}=\ldots=c_{n+k}=0$ but $\gamma\left(K_{0} \cap E_{1}^{\perp} \cap \partial B_{r}\right) \leqslant k-1$. Denote

$$
B=E_{1}^{\perp} \cap \partial B_{r} \backslash\left(K_{0} \cap E_{1}^{\perp} \cap \partial B_{r}\right), A=\partial D_{m}=E_{2} \cap \partial B_{R} .
$$

From (3.4) we see that $-f(x) \geqslant 0$ on $A$ and $-f(x) \leqslant 0$ on $B$. Since $B \cap K_{0}=\emptyset$ and $B$ is bounded, by Lemma 2.4 we can find $\varepsilon>0$ and an odd homeomorphism $\eta$ of $E$ onto itself such that

$$
\eta x=x \text { on } A \text { and }-f(\eta x) \leqslant-\varepsilon \text { on } B .
$$

Since $c_{n+k}=0$ we can find $B_{0} \in \Gamma_{n+k}$ such that

$$
f(x) \leqslant(1 / 2) \varepsilon \text { on } B_{0} .
$$

Let $\widetilde{B}=\eta^{-1} B_{0}$. Then from $3^{\circ}$ we know $\widetilde{B} \in \Gamma_{n+k}$, and hence $\widetilde{B} \cap B \neq \emptyset$ by $5^{\circ}$. Suppose $x_{0} \in \widetilde{B} \cap B$. Then it follows from $x_{0} \in B$ and (3.6) that $f\left(\eta x_{0}\right) \geqslant \varepsilon$. On the 
other hand, since $\eta x_{0} \in B_{0}, f\left(\eta x_{0}\right) \leqslant(1 / 2) \varepsilon$ by (3.7). This contradiction proves $6^{\circ}$. The proof of the theorem is now complete.

Theorem 3.4. In Theorem 3.3, if we replace (3.4) by

$$
f(x) \geqslant 0 \text { on } \partial B_{\rho} \cap E_{2} \text { and } f(x) \leqslant R \text { on } E_{1}^{\perp}
$$

for some positive numbers $\rho$ and $R$, then the same result holds.

Proof: Denote $\Sigma_{k}=\{A \in \Sigma: \gamma(A) \geqslant k\}$ and let

$$
c_{k}=\sup _{A \in \Sigma_{k}} \inf _{x \in A} f(x), k=n+1, \ldots, m .
$$

Since $\gamma\left(E_{2} \cap \partial B_{\rho}\right)=m$, we know by (3.8) that $c_{m} \geqslant 0$. For any $A \in \Sigma_{n+1}$, $A \cap E_{1}^{\perp} \neq \emptyset$. Hence it follows from (3.8) that $\inf _{x \in A} f(x) \leqslant R, c_{n+1} \leqslant R$ and so, since $\Sigma_{k+1} \subset \Sigma_{k}$, we get

$$
R \geqslant c_{n+1} \geqslant \ldots \geqslant c_{m} \geqslant 0 .
$$

Now to prove the theorem it suffices to prove

(i) if $c_{n+k}=\ldots=c_{m}=0,1 \leqslant k \leqslant m-n$, then $\gamma\left(K_{0} \cap E_{2} \cap \partial B_{\rho}\right) \geqslant$ $m-n-k+1$

(ii) if $c_{n+k_{1}}=\ldots=c_{n+k_{2}}=c>0,1 \leqslant k_{1} \leqslant k_{2} \leqslant m-n$, then $\gamma\left(K_{c}\right) \geqslant$ $k_{2}-k_{1}+1$.

As in the proof of Theorem 3.3, we only prove (i).

Suppose by contradiction that $\gamma\left(K_{0} \cap E_{2} \cap \partial B_{\rho}\right) \leqslant m-n-k$. Then since $c_{m}=0$ and $E_{2} \cap \partial B_{\rho} \in \Sigma_{m}$, we have $\inf _{x \in E_{2} \cap \theta B_{\rho}} f(x) \leqslant 0$, which together with (3.8) implies $\inf _{x \in E_{2} \cap \theta B_{\rho}} f(x)=0$. Choose a symmetric open neighbourhood $N$ of the compact set $K_{0} \cap E_{2} \cap \partial B_{\rho}$ such that $\gamma(\bar{N}) \leqslant m-n-k$. Then $\gamma\left(E_{2} \cap \partial B_{\rho} \backslash N\right) \geqslant m-(m-n-k)=$ $n+k$.

Let $S=E_{2} \cap \partial B_{\rho} \backslash N$. Then $S$ is a symmetric bounded closed set and $-f(x) \leqslant 0$ on $S, S \cap K_{0}=0$. By Lemma 2.4 we can find some $\varepsilon>0$ and an odd homeomorphism $\eta$ of $E$ onto $E$ such that

$$
-f(\eta x) \leqslant-\varepsilon \text { on } S .
$$

On the other hand, $\eta(S) \in \Sigma$ and $\gamma(\eta(S)) \geqslant \gamma(S) \geqslant n+k$. Therefore $\eta(S) \in \Sigma_{n+k}$, and hence $c_{n+k} \geqslant \varepsilon>0$ by (3.9), which contradicts $c_{n+k}=0$. The proof is complete.

\section{Nontrivial Soldtions of a Resonance Problem}

In this section, we apply the results of Section 3 to the resonance problem (1.3). For the nonlinear term $p(x, t)$, let $P(x, t)=\int_{0}^{t} p(x, s) d s$; we consider the following conditions:

$(I)_{m} \quad P(x, t) \geqslant(1 / 2)\left(\lambda_{m}-\lambda_{k}\right) t^{2}$ for $|t|$ sufficiently small. 
$(I I)_{m} \quad P(x, t) \leqslant(1 / 2)\left(\lambda_{m+1}-\lambda_{k}\right) t^{2}$ for all $t$ in $R^{1}$.

(III) $p$ is bounded, $\lim _{|t| \rightarrow \infty} P(x, t)=-\infty$.

(IV) $\lim _{|t| \rightarrow \infty} t p(x, t)=0, \lim _{|t| \rightarrow \infty} P(x, t)=A(x), A(x) \in C(\bar{\Omega})$.

(V) $p(x,-t)=-p(x, t)$.

In conditions (III) and (IV), we suppose that the limits are uniform for $x \in \bar{\Omega}$.

For $u \in E=W_{0}^{1,2}(\Omega)$, let

$$
I(u)=-(1 / 2) \int_{\Omega}\left(|D u|^{2}-\lambda_{k} u^{2}\right) d x+\int_{\Omega} P(x, u(x)) d x .
$$

It is well-know that under certain growth conditions on $p, I$ is well defined and that critical points of $I$ are solutions of (1.3). Since $p(x, 0)=0,0$ is a trivial critical point. We are looking for nontrivial critical points of $I$. Conditions (III) and (IV) are sufficient restrictions on the growth of $p$. Moreover, condition (III) guarantees that $I$ satisfies the (PS) condition on $E$ (see [13]) while condition (IV) is a "strong" resonance condition, in which case $I$ does not satisfy the (PS) condition but verifies condition (C) (see [1]). Conditions $(I)_{m}$ and $(I I)_{m}$ correspond to the boundary conditions in the critical point theorems and in this case $I$ satisfies the relaxed boundary condition (1.2) but not (1.1). We are going to prove the following theorems.

Theorem 4.1. If $p$ satisfies for some $m \geqslant k$ the conditions $(I)_{m},(I I)_{m}$ and (III), then (1.3) has at least one nontrivial solution.

Theorem 4.2. If $p$ satisfies for some $m \geqslant k+1$ the conditions $(I)_{m},(I I)_{m}$ and (IV), then (1.3) has at least one nontrivial solution.

TheOREM 4.3. Suppose $p$ satisfies for some $m \geqslant k$ the conditions $(I)_{m},(I I)$ or (IV), and (V). Then problem (1.3) has at least $\Sigma_{i=h}^{m} m\left(\lambda_{i}\right)$ pairs of nontrivial solutions.

The above theorems are related to some known results. When $m=k$, Theorem 4.1 is in some sense a dual version of Theorem $1(b)(\beta)$ of Thews [14]. The case Thews considered essentially implies $P(x, t)<0$ for $t$ small, $\lim _{|t| \rightarrow \infty} P(x, t)=+\infty$ and $P(x, t)<(1 / 2)\left(\lambda_{k+1}-\lambda_{k}\right) t^{2}$ for all $t$. Theorem 4.2 is related to some results of Bartolo, Benci and Fortunato [1], but they require $P(x, t)<0$ for $t$ small too. Another related result of Theorem 4.2 is Theorem 1 of Capozzi, Lupo and Solimini [4], where they do not require $(I I)_{m}(m \geqslant k+1)$, but they assume $p(x, t)=p(t)$ and $p^{\prime}(0)<\lambda_{k+1}-\lambda_{k}$ which is more restrictive than $(I)_{k+1}$. Theorem 4.3 is a slight generalisation of Theorem 2.22 of Rabinowitz [13]; it also relates to a result in Bartolo, Benci and Fortunato [1], but their condition implies $P(x, t)<-\left(\lambda_{k}-\lambda_{k}\right) t^{2}$ for some integer $h \leqslant k$.

Proof of Theorem 4.1: 
Let $E=W_{0}^{1,2}(\Omega)$ with norm $\|u\|^{2}=\int_{\Omega}|D u|^{2} d x$, and suppose $I$ is defined by (4.1). For $u \in E$, define

$$
J(u)=\int_{\mathbf{n}} P(x, u(x)) d x .
$$

It is easy to see that $I^{\prime}(u)=-u+D u$, and $D: E \rightarrow E$ is completely continuous. Moreover, it follows from (III) that $I$ satisfies the (PS) condition on $E$ (see [13]).

Let $E^{(i)}$ denote the eigenspace corresponding to the eigenvalue $\lambda_{i}$ and denote

$$
E_{1}=\bigoplus_{i \leqslant m} E^{(i)}, \text { and } E_{2}=\bigoplus_{i \geqslant m+1} E^{(i)} .
$$

Then $E=E_{1} \oplus E_{2}, E_{1}$ is finite dimensional, $E_{2}$ is infinite dimensional.

Let $v_{m} \in E^{(m)} \cap \partial B_{1}$. We are going to prove that there exist $\varepsilon, R, 0<\varepsilon<R$, and $M>0$ such that for

$$
S=E_{1} \cap \partial B_{6}, \text { and } Q=\left\{s v_{m}: 0 \leqslant s \leqslant R\right\} \oplus E_{2} \cap B_{R},
$$

the following conclusions hold:

$$
\begin{aligned}
& 1^{\circ} \cdot I(u) \geqslant 0 \text { on } S \\
& 2^{\circ} \cdot I(u) \leqslant 0 \text { on } \partial Q ; \\
& 3^{\circ} \cdot I(u) \leqslant M \text { on } Q .
\end{aligned}
$$

If $1^{\circ}-3^{\circ}$ in (4.2) hold true, then by Theorem 3.1 or Theorem 3.2 we know that I has a critical value $c \geqslant 0$ corresponding to a nontrivial critical point, and hence (1.3) has a nontrivial solution as required.

For $u \in E_{1}$, we have

$$
I(u)=-(1 / 2) \int_{\Omega}\left(|D u|^{2}-\lambda_{k} u^{2}\right) d x+J(u) \geqslant-(1 / 2)\left(1-\lambda_{k} / \lambda_{m}\right)\|u\|^{2}+J(u) .
$$

Since $E_{1}$ is finite dimensional, $u \in E_{1}$ small in norm implies $\underset{x \in \Omega}{\max }|u(x)|$ is small. Therefore by $(I)_{m}$ we can find $\varepsilon>0$ sufficiently small such that for $u \in E_{1} \cap \partial B_{\varepsilon}$,

$$
\begin{gathered}
P(x, u(x)) \geqslant(1 / 2)\left(\lambda_{m}-\lambda_{k}\right) u^{2}(x), \\
J(u) \geqslant(1 / 2)\left(\lambda_{m}-\lambda_{k}\right) \int_{\Omega} u^{2}(x) d x \geqslant(1 / 2)\left(1-\lambda_{k} / \lambda_{m}\right)\|u\|^{2} .
\end{gathered}
$$

Thus $I(u) \geqslant-(1 / 2)\left(1-\lambda_{k} / \lambda_{m}\right)\|u\|^{2}+J(u) \geqslant 0$, and $1^{\circ}$ is satisfied.

Denote

$$
Q_{1}=\left\{s v_{m}: 0 \leqslant s \leqslant R\right\} \oplus E_{2} \cap \partial B_{R}, \quad Q_{2}=B_{R} \cap E_{2}, \quad Q_{s}=\left\{R v_{m}\right\} \oplus B_{R} \cap E_{2} .
$$


Clearly $\delta Q=Q_{1} \cup Q_{2} \cup Q_{3}$.

By $(I I)_{m}$ we get for $u \in E_{2}$ that

$$
\begin{aligned}
I(u) & =-(1 / 2) \int_{\Omega}\left(|D u|^{2}-\lambda_{k} u^{2}\right) d x+J(u) \\
& \leqslant-(1 / 2)\left(1-\lambda_{k} / \lambda_{m+1}\right)\|u\|^{2}+1 / 2\left(\lambda_{m+1}-\lambda_{k}\right) \int_{\Omega} u^{2} d x \leqslant 0 .
\end{aligned}
$$

By (III) we get

$$
\lim _{R \rightarrow \infty} J\left(R v_{m}\right)=-\infty
$$

Now for $u \in Q_{3}$, suppose $u=R v_{m}+v, v \in B_{R} \cap E_{2}$. By the boundedness of $p$ and (4.4) we obtain

$$
\begin{aligned}
I(u) & =-1 / 2 \int_{\Omega}\left(|D v|^{2}+R^{2}\left|D v_{m}\right|^{2}-\lambda_{k} v^{2}-R^{2} \lambda_{k} v_{m}^{2}\right) d x+J\left(R v_{m}+v\right) \\
& \leqslant-1 / 2 \int_{\Omega}\left(|D v|^{2}-\lambda_{k} v^{2}\right) d x+J\left(R v_{m}\right)+J\left(R v_{m}+v\right)-J\left(R v_{m}\right) \\
& \leqslant-1 / 2\left(1-\lambda_{k} / \lambda_{m+1}\right)\|v\|^{2}+J\left(R v_{m}\right)+\int_{\Omega} \int_{R v_{m}}^{R v_{m}+v} p(x, t) d t d x \\
& \leqslant-1 / 2\left(1-\lambda_{k} / \lambda_{m+1}\right)\|v\|^{2}+\max |p(x, t)| \int_{\Omega}|v| d x+J\left(R v_{m}\right) \\
& \leqslant-1 / 2\left(1-\lambda_{k} / \lambda_{m+1}\right)\|v\|^{2}+M_{1}\|v\|+J\left(R v_{m}\right) \rightarrow-\infty(R \rightarrow \infty) .
\end{aligned}
$$

For $u \in Q_{1}$, suppose $u=s v_{m}+v, v \in \partial B_{R} \cap E_{2}, 0 \leqslant s \leqslant R$. Then

$$
\begin{aligned}
I(u) & \leqslant-1 / 2 \int_{\Omega}\left(|D v|^{2}-\lambda_{k} v^{2}\right) d x+J\left(s v_{m}+v\right) \\
& \leqslant-1 / 2\left(1-\lambda_{k} / \lambda_{m+1}\right)\|v\|^{2}+\max |p(x, t)| \int_{\Omega}\left(|v|+s\left|v_{m}\right|\right) d x \\
& \leqslant-1 / 2\left(1-\lambda_{k} / \lambda_{m+1}\right) R^{2}+M_{2} R \rightarrow-\infty(R \rightarrow \infty) .
\end{aligned}
$$

From (4.5), (4.6) and (4.3) we see that $2^{\circ}$ in (4.2) is satisfied.

For $u \in Q$, suppose $u=s v_{m}+v, 0 \leqslant s \leqslant R, v \in B_{R} \cap E_{2}$. Then

$$
\begin{aligned}
I(u) & \leqslant-1 / 2 \int_{\Omega}\left(|D v|^{2}-\lambda_{k} v^{2}\right) d x+J\left(v+s v_{m}\right) \leqslant J\left(v+s v_{m}\right) \\
& \leqslant \max |p(x, t)| \int_{\Omega}\left(|v|+s\left|v_{m}\right|\right) d x \leqslant M_{2} R .
\end{aligned}
$$

Hence $3^{\circ}$ is satisfied. The proof is complete. 
Let us make a few remarks before giving proofs of the other theorems.

First, from the proof of Theorem 4.1 we see that the conditions in this theorem can be replaced by:

$(I)_{m}^{\prime} \quad \int_{\Omega} P(x, u(x)) d x \geqslant 1 / 2\left(\lambda_{m}-\lambda_{k}\right) \int_{\Omega} u(x)^{2} d x$ for $u \in \partial B_{c} \cap E_{1}, e$ small;

$(I I)_{m}^{\prime} \quad \int_{\Omega} P(x, u(x)) d x \leqslant 1 / 2\left(\lambda_{m+1}-\lambda_{h}\right) \int_{\Omega} u(x)^{2} d x$ for $u \in E_{2}$;

$(I I I)^{\prime} \quad p$ is bounded, $\int_{\Omega} P(x, u(x)) d x \rightarrow-\infty$ for $u \in E^{(m)}$ and $\|u\| \rightarrow \infty$.

Second, in the proof of Theorem 4.1, since $0 \in \partial Q$, we must have

$$
\sup _{x \in O Q} I(u)=0 .
$$

Furthermore, since $S$ is compact, one can easily check that

$$
\inf _{u \in S} I(u)>0
$$

if and only if $(I)_{m}^{\prime}$ is satisfied and

$$
\int_{\Omega} P(x, u(x)) d x>1 / 2\left(\lambda_{m}-\lambda_{k}\right) \int_{\Omega} u^{2} d x \text { for all } u \in \partial B_{c} \cap E^{(m)}
$$

Therefore, in this case, the relaxed boundary condition provides a better result than the original one.

Proof of Theorem 4.2::

Let $I, E, S, Q$ be defined as in the proof of Theorem 4.1. Now $I$ satisfies condition (C) by (IV) (see [1]). Since $S$ is bounded, from Theorem 3.2 we see that to complete the proof it suffices to prove $1^{\circ}-3^{\circ}$ in (4.2).

It follows from (IV) that $p$ is bounded, and so by $(I)_{m},(I I)_{m}$ and the proof of Theorem 4.1 we see that $1^{\circ}, 3^{\circ},(4.3)$ and (4.6) are all satisfied.

For $u \in Q_{3}$,

$$
\begin{aligned}
I(u) & =-1 / 2 \int_{\Omega}\left(|D u|^{2}-\lambda_{k} u^{2}\right) d x+J(u) \\
& \leqslant-1 / 2\left(1-\lambda_{k} / \lambda_{k+1}\right)\|u\|^{2}+M\|u\| \rightarrow-\infty(R \rightarrow \infty) .
\end{aligned}
$$

This together with (4.3) and (4.6) shows $2^{\circ}$ in (4.2) is satisfied. And the proof is complete.

Proof of TheOREM 4.3:

Let $I, E$ and $E^{(i)}$ be defined as in the proof of Theorem 4.1. Define

$$
E_{1}=\bigoplus_{i \leqslant k-1} E^{(i)}, E_{2}=\bigoplus_{i \leqslant m} E^{(i)}
$$


Then $\operatorname{dim} E_{2}-\operatorname{dim} E_{1}=\Sigma_{i=k}^{m} m\left(\lambda_{i}\right)$.

Since $p$ satisfies (III) or (IV), $I$ satisfies condition (C) and so by Theorem 3.4 we need only prove that for some positive numbers $\rho$ and $R$,

$$
I(u) \geqslant 0 \text { on } \partial B_{\rho} \cap E_{2} \text { and } I(u) \leqslant R \text { on } E_{1}^{\perp} .
$$

By $(I)_{m}$ and the proof of Theorem 4.1, there exists $\rho>0$ sufficiently small such that $I(u) \geqslant 0$ for $u \in \partial B_{\rho} \cap E_{2}$.

Let

$$
E_{3}=\bigoplus_{i \geqslant k+1} E^{(i)}
$$

Then $E_{1}^{\perp}=E^{(k)} \oplus E_{3}$. For $u \in E_{1}^{\perp}$, suppose $u=v_{k}+v, v_{k} \in E^{(k)}, v \in E_{3}$. Then

$$
\begin{aligned}
I(u) & =-1 / 2 \int_{\Omega}\left(|D v|^{2}-\lambda_{k} v^{2}\right) d x+J\left(v_{k}+v\right) \\
& \leqslant-1 / 2\left(1-\lambda_{k} / \lambda_{k+1}\right)\|v\|^{2}+J\left(v_{k}\right)+\left(J\left(v_{k}+v\right)-J\left(v_{k}\right)\right) \\
& \leqslant-1 / 2\left(1-\lambda_{k} / \lambda_{k+1}\right)\|v\|^{2}+J\left(v_{k}\right)+M\|v\| \\
& \leqslant M_{1}+\sup _{E^{(k)}} J\left(v_{k}\right)
\end{aligned}
$$

where $M, M_{1}$ are constants independent of $u$.

From (IV) we can easily prove that $J$ is bounded on $E^{(k)}$ and hence it follows from (4.7) that there exists $R$ such that $I(u) \leqslant R$ on $E_{1}^{\perp}$. The proof is complete.

\section{REFERENCES}

[1] P. Bartolo, V. Benci and P. Fortunato, 'Abstract critical point theorems and applications to some nonlinear problems with "strong" resonance at infinity', Nonlinear Anal. 7 (1983), 981-1012.

[2] V. Benci and P.H. Rabinowitz, 'Critical point theorems for indefinite functionals', Invent. Math 52 (1979), 241-273.

[3] K.C. Chang, Critical point theory and its applications (Shanhai Sci. and Tech. Press, 1986).

[4] A. Capozzi, D. Lupo and S. Solimini, 'On the existence of a nontrivial solution to nonlinear problems at resonance', Nonlinear Anal. 13 (1989), 151-163.

[5] Y. Du, 'A deformation lemme and some critical point theorems', Bull. Austral. Math. Soc. 43 (1991), 161-168.

[6] N. Ghoussoub, 'A minimax principle with a relaxed boundary condition', Proc. Amer. Math. Soc (to appear).

[7] N. Ghoussoub, 'Location, multiplicity and Morse indices of min-max critical points', (preprint). 
[8] N. Ghoussoub and D. Preiss, 'A mountain pass principle for locating and classifying critical points', A.I.H.P.-Analyse non lineaire 6 (1989), 321-330.

[9] D. Guo, J. Sun and G. Qi, 'Some extensions of the mountain pass lemma', Differential Integral Equations 1 (1988), 351-358.

[10] P. Pucci and J. Serrin, 'Extensions of the mountain pass lemma', J. Funct. Anal. 59 (1984), 185-210.

[11] P. Pucci and J. Serrin, 'A mountain pass lemma', J. Differential Equations 60 (1985), 142-149.

[12] P.H. Rabinowitz, Minimax methods in critical point theory with applications to differential equations, CBMS Regional Conf. Ser. in Math 65 (Amer. Math. Soc., Providence, RI, 1986).

[13] P.H. Rabinowitz, 'Some minimax theorems and applications to nonlinear partial differential equations', in Nonlinear Analysis, (L. Cesari, R. Kannan and H.F. Weinberger, Editors) (Academic Press, New York, 1978), pp. 161-177.

[14] K. Thews, 'A reduction method for some nonlinear Dirichlet problems', Nonlinear Anal. 3 (1979), 795-813.

Department of Mathematics, Statistics and Computing Science

University of New England

Armidale NSW 2351

Australia 